\title{
VALUES OF ENTIRE FUNCTIONS REPRESENTED BY GAP DIRICHLET SERIES
}

\section{F. SUNYER I BALAGUER}

Introduction. In two previous notes [9], ${ }^{1}$ I stated some results which, in a general way, may be expressed as follows: If the Taylor series which represents an entire function satisfies a certain gap condition (which depends only on the order of $F(z)$ ), the zeros of $F(z)-f(z)$ are not exceptional with respect to the proximate order of $F(z)$ by any meromorphic function $f(z) \not \equiv \infty$ of lower order.

On the other hand, Mandelbrojt (see for instance [3, Theorem XXV]) proves that an entire function represented by a Dirichlet series takes each value $a \neq \infty$, except at most one, in any horizontal strip of width greater than a quantity which depends only on the order $(R)$ and on the upper density of the sequence of exponents of the series.

In the present note we shall prove some results closely related to those of my above mentioned notes and to that of Mandelbrojt just quoted. These results may briefly be stated as follows: If an entire function $F(s)$ can be represented by a Dirichlet series satisfying certain gap conditions, the zeros of $F(s)-a$ cannot be exceptional with respect to the proximate order $(R)$ of $F(s)$ in any strip of width greater than a quantity, determined by the order, for every value of $a \neq \infty$ without exception.

In $\$ 1$ we shall deal with functions of finite order $(R)$; in $\$ 2$, we shall give results concerning functions of infinite order $(R)$.

I think it will be of interest to remark that the theorems of the type of the well known Hadamard's theorem (which asserts that a Taylor series satisfying a specific gap condition cannot be continued analytically outside its circle of convergence) together with the results concerning relationship between gap properties and the position of the Julia lines, and again together with the results of my above mentioned notes and those contained in one of my papers [10], enable us to state the following general principle (without pretending that it holds for every case). By means of gap conditions in the Taylor series the disappearance of the possibility of the existence of the exceptional cases can be affirmed.

Therefore, the content of this paper may be regarded as a link

Presented to the Society, February 24, 1951; received by the editors January 9, 1951 and, in revised form, June 20, 1952.

${ }^{1}$ Numbers in brackets refer to the bibliography at the end of the paper. 
extending this general principle to the Dirichlet series.

Finally, I wish to thank our colleague J. Savé for the help he has given me when writing this work in English.

1.1. First of all I want to fix the meaning of a notation which will continuously be used. By $\epsilon(\sigma)$ will always be represented a positive quantity tending to zero when the variable (represented here by $\sigma$ ) tends to $-\infty$; but it must be borne in mind that, even in one formula, the notation $\epsilon(\sigma)$ may represent different quantities, with only the common properties of being positive and that: $\lim _{\sigma=-\infty} \epsilon(\sigma)=0$. It is evident, therefore, that $\epsilon(\sigma)$ means almost the same as $o(1)$, but we have introduced the notation $\epsilon(\sigma)$ in order to show the variable and the limit to which it tends.

According to the custom the sequence $\left\{\lambda_{n}\right\}$ will have, throughout the present paper, the following properties:

$$
0=\lambda_{0}<\lambda_{1}<\cdots<\lambda_{n}<\cdots, \quad \lim _{n=\infty} \lambda_{n}=\infty .
$$

Let $N(\lambda)$ be the greatest of the $n$ such that $\lambda_{n}<\lambda$. Following Mandelbrojt [4], we shall call $D(\lambda)=N(\lambda) / \lambda$ the density function of $\left\{\lambda_{n}\right\}$, and the quantity $D^{*}=\lim \sup _{\lambda=\infty} D(\lambda)$ shall be called the upper density of $\left\{\lambda_{n}\right\}$. On the other hand, Mandelbrojt similarly introduces the function $\bar{D}(\lambda)=\lambda^{-1} \int_{0}^{\lambda} D(x) d x$ which is called the mean density function, and $\bar{D}^{*}=\lim \sup _{\lambda=\infty} \bar{D}(\lambda)$ which is called the mean upper density of $\left\{\lambda_{n}\right\}$.

Let $f(s)$ be an entire function satisfying the following relationship:

$$
\underset{-\infty<t<+\infty}{\text { l.u.b. }}|f(\sigma+i t)|=M(\sigma, f)<\infty
$$

for any real value of $\sigma$ (in particular, every function represented by a Dirichlet series absolutely convergent in the whole plane will have this property). For that type of function Ritt ${ }^{2}$ defines the order (which for that reason is called order $(R)$ ) in the following way:

$$
\rho=\limsup _{\sigma=-\infty} \frac{\log _{2} M(\sigma, f)}{-\sigma} \quad\left(\log _{2} X=\log \log X\right)
$$

will be called the order $(R)$ of $f(s)$; we shall also express it by saying

$2 \mathrm{~J}$. F. Ritt, On certain points in the theory of Dirichlet series, Amer. J. Math. vol. 50 (1928) pp. 73-86. I could not possibly consult this paper; all I know of it is the part referred to by Mandelbrojt [3]. This is the reason for not including it in the bibliography. 
that $f(s)$ is of order $(R)$ equal to $\rho$.

Likewise, representing by $Y$ the horizontal strip $\theta_{1} \leqq t \leqq \theta_{2}$ and putting

$$
M(\sigma, Y, f)=\underset{\theta_{1} \leqq t \leqq \theta_{\mathbf{2}}}{\text { l.u.b. }}|f(\sigma+i t)|,
$$

the order $(R)$ of $f(s)$ in $Y$ will be

$$
\rho=\limsup _{\sigma=-\infty} \frac{\log \log ^{+} M(\sigma, Y, f)}{-\sigma},
$$

where $\log ^{+} X=\log X$ if $X \geqq 1$ and $\log ^{+} X=0$ if $X \leqq 1$.

As in the theory of entire functions where the notion of order with respect to the modulus of the variable is improved by the introduction of proximate orders, we shall similarly introduce the notion of proximate orders $(R)$. We shall give the following definition: The function $\rho(\sigma)$ will be a proximate order $(R)$ if

$$
\lim _{\sigma=-\infty} \rho(\sigma)=\rho, \quad \lim _{\sigma=-\infty} \rho^{\prime}(\sigma) \sigma=0,
$$

and then the function $f(s)$ will be of proximate order $(R) \rho(\sigma)$ if

$$
\limsup _{\sigma=-\infty} \frac{\log M(\sigma, f)}{V(\sigma)}=1 \quad\left(V(\sigma)=e^{-\rho(\sigma) \sigma}\right),
$$

and, likewise, the function $f(s)$ will be, in the strip $Y$, of proximate order $(R) \rho(\sigma)$ if

$$
\limsup _{\sigma=-\infty} \frac{\log M(\sigma, Y, f)}{V(\sigma)}=1
$$

As in the present note we shall deal only with orders $(R)$; in the following lines the $R$ will be dropped and the orders simply called order and proximate order.

1.2. We shall represent by $n(x, Y, f)$ the number of zeros of $f(s)$ situated in the part $0 \geqq \sigma \geqq x$ of the strip $Y$; with this notation we shall state-and later on prove-the following result:

THEOREM I. Let

$$
F(s)=\sum_{n=0}^{\infty} a_{n} e^{-\lambda_{n}}
$$

be a function, represented by a Dirichlet series convergent in the whole

3 Since in this work we shall speak only of horizontal strips, we shall henceforth suppress the word "horizontal" which will always be understood. 
plane, of order $\rho$ and of proximate order $\rho(\sigma)$; given a strip $Y$ of width $>\pi / \rho$, there exists a number $\Delta$ depending only on $Y, \rho$, and $h$ such that if, on denoting by $\bar{D}^{*}$ the mean upper density of $\left\{\lambda_{n}\right\}$,

$$
\bar{D}^{*}<\Delta \text { and } \liminf _{n=\infty}\left(\lambda_{n+1}-\lambda_{n}\right)=h>0,
$$

then for any given finite value of a (without exception)

$$
\limsup _{\sigma=-\infty} \frac{n(\sigma, Y, F-a)}{V(\sigma)} \geqq B_{0}>0,
$$

where $B_{0}$ depends only on $\rho, Y$, and $h$.

1.3. The proof of this theorem is based on the following lemma:

LEMMA 1. Let $f(s)$ be a holomorphic function in the strip $Y\left(\theta_{1} \leqq t \leqq \theta_{2}\right)$ if

(i) $f(s)$ is in $Y$ of order $\rho>\pi /\left(\theta_{2}-\theta_{1}\right)$ and of proximate order $\rho(\sigma)$,

(ii) in a strip $Y^{\prime}$, contained in $Y$,

$$
\limsup _{\sigma=-\infty} \frac{\log M\left(\sigma, Y^{\prime}, f\right)}{V(\sigma)} \geqq A>0,
$$

then there exists a quantity $B_{0}$ which depends only on $Y, Y^{\prime}, \rho$, and $A$, such that, if

$$
\limsup _{\sigma=-\infty} \frac{n(\sigma, Y, f)}{V(\sigma)}<B_{0},
$$

there will be a sequence $\left\{s_{n}=\sigma_{n}+i t_{n}\right\}$ (lim $\left.\sigma_{n}=-\infty\right)$ of points contained in $Y$ and a quantity $r$ depending on $Y, Y^{\prime}$, and $A$, such that in the circles $\left|s-s_{n}\right| \leqq r$ the inequality

$$
\log |f(s)|<0
$$

holds.

First of all, without diminishing the generality, we can suppose that $\rho\left(\theta_{2}-\theta_{1}\right) / \pi$ is not an integer. Furthermore, we may also suppose that the strip $Y$ is $|t| \leqq \pi / 2$, that $Y^{\prime}$ is $|t| \leqq \beta$, and that $\rho$ is not an integer and is $>1$.

We shall write

$$
\psi(s)=\phi\left(e^{-s}\right),
$$

where $\phi(z)$ is the canonical product of Weierstrass which has for zeros the points $z_{n}=e^{-s n}$, where the $\left\{s_{n}\right\}$ represent the zeros of $f(s)$ situated in the part $\sigma \leqq 0$ of $Y$. 
If

$$
\limsup _{\sigma=-\infty} \frac{n(\sigma, Y, f)}{V(\sigma)}=B,
$$

according to the properties of the canonical products it follows easily that the inequality

$$
\log M(\sigma, Y, \psi)<(1+\epsilon(\sigma)) C B V(\sigma)
$$

holds, where $C$ depends only on $\rho$. Consequently

$$
\limsup _{\sigma=-\infty} \frac{\log M\left(\sigma, Y^{\prime}, f / \psi\right)}{V(\sigma)} \geqq A-C B .
$$

Furthermore let $Y_{1}\left(|t| \leqq \beta_{1}\right)$ be a strip such that $\beta_{1}>\beta$ and $\beta_{1} \rho>\pi / 2$, for instance

$$
2 \beta_{1}=\frac{\pi}{2}+\max \left(\beta, \frac{\pi}{2 \rho}\right),
$$

then it follows that the inequality (we suppose $B<A / C$ )

$$
\limsup _{\sigma=-\infty} \frac{\log M\left(\sigma, Y_{1}, \psi / f\right)}{V(\sigma)}>K(A-C B)
$$

holds, where $K$ depends only on $Y_{1}, Y^{\prime}$, and $\rho$. In order to prove this latter inequality we shall have to take into account that $f(s) / \psi(s)$ has no zeros in the part $\sigma \leqq 0$ of $Y$.

By aid of a lemma of Bernstein [11, Lemma II] slightly modified for being valid in the strips, and applying it to $\psi(s)$, it is possible to prove that in the strip $Y_{2}\left(|t| \leqq \beta_{2}=\left(\pi+2 \beta_{1}\right) / 4\right)$ the relationship

$$
\limsup _{\sigma=-\infty} \frac{\log M\left(\sigma, Y_{2}, f / \psi\right)}{V(\sigma)}<K_{1}+K_{2} B
$$

will hold, where $K_{1}$ and $K_{2}$ depend only on $Y_{2}$ and $\rho$.

From the inequality (1.3.1) follows the existence of a sequence of points $\left\{s_{n}^{\prime}\right\}$ in which

$$
\log \left|f\left(s_{n}^{\prime}\right) / \psi\left(s_{n}^{\prime}\right)\right| \leqq-\frac{1}{2} K(A-C B) V\left(\sigma_{n}^{\prime}\right),
$$

and consequently in the circles

$$
\left|s-s_{n}^{\prime}\right| \leqq \beta_{2}-\beta_{1}
$$

there exists a domain, inside of which the point $s_{n}^{\prime}$ will be found and which will have points in common with the circumference 


$$
\left|s-s_{n}^{\prime}\right|=\beta_{2}-\beta_{1}
$$

in which

$$
\log |f(s) / \psi(s)| \leqq-\frac{1}{2} K(A-C B) V\left(\sigma_{n}^{\prime}\right) .
$$

From this result and from (1.3.2) may be deduced, by aid of a theorem of Milloux $^{4}$ (if, for instance, $2 B<A / C$ ), the existence of a quantity $r$ which depends only on $\rho, Y^{\prime}$, and $A$, such that in the circles $\left|s-s_{n}^{\prime}\right| \leqq r$ the inequality

$$
\log |f(s) / \psi(s)| \leqq-\frac{1}{3} K(A-C B) V\left(\sigma_{n}^{\prime}\right)
$$

will hold, and consequently

$$
\log |f(s)|<-\frac{1}{3} K(A-C B) V\left(\sigma_{n}^{\prime}\right)+\left(1+\epsilon\left(\sigma_{n}^{\prime}\right)\right) K_{s} C B V\left(\sigma_{n}^{\prime}\right),
$$

where $K_{3}$ depends only on $\rho, Y^{\prime}$, and $A$.

Finally if $B$ is smaller than a quantity $B_{0}$ which depends only on $Y^{\prime}, \rho$, and $A$, in the same circles the inequality

$$
\log |f(s)|<0
$$

will hold, which is the result we had to prove.

1.4. Now we shall prove Theorem I. In order to simplify we shall suppose that the strip $Y$ is $|t| \leqq \pi / 2$ and that, according to the hypothesis of the theorem, $\rho>1$; this does not restrict the generality of the proof, because it is evident that it may be attained by means of linear transformation upon the variable $s$. Moreover, it is interesting to point out that, since $D^{*}<1 / h$, the series which represents $F(s)$ will be absolutely convergent in the whole plane, since, according to a well known result, a Dirichlet series whose exponents form a sequence of finite upper density has its abscissa of convergence equal to its abscissa of absolute convergence; therefore, we are concerned with a case in which the definitions of order of $F(s)$ are possible.

According to a result of Mandelbrojt [4, Theorem a] for any $s_{0}$ $=\sigma_{0}+i t_{0}$, inside the circle

$$
\left|s-s_{0}\right| \leqq \pi \bar{D}^{*}+\epsilon,
$$

where $\epsilon$ is a given positive quantity, arbitrarily small, but fixed, there will exist a point $s^{\prime}$ at which

$$
\log \left|F\left(s^{\prime}\right)\right|>\log \left|a_{n}\right|-\lambda_{n} \sigma_{0}-\log \left(\lambda_{n} \Lambda_{n}^{*}\right)-c_{\epsilon},
$$

4 For a very precise statement of this theorem see [10, Lemmas 1,4] and for the proof see [6, pp. 96-100].

- When the Dirichlet series contains a constant term (when $a_{0} \neq 0$ ), the definition of the $\Lambda(r)$ (and, therefore, of the $\Lambda_{n}^{*}$ ) given by Mandelbrojt must vary slightly. 
where $c_{\epsilon}$ is a constant which depends on $\epsilon$; and this inequality will hold for every value of $\boldsymbol{n}$.

On the other hand, according to a result contained in the same paper of Mandelbrojt [4, p. 355] for values of $\boldsymbol{n}$ sufficiently large

$$
\frac{-\log \left(\lambda_{n} \Lambda_{n}^{*}\right)-c_{\epsilon}}{\lambda_{n}}>-D^{*}\left(7-3 \log \left(h D^{*}\right)\right)-\epsilon .
$$

Let us write

$$
\log \mu(\sigma)=\max _{1 \leqq n<\infty}\left(\log \left|a_{n}\right|-\lambda_{n} \sigma\right) ;
$$

then evidently, if $\sigma$ is smaller than a certain negative quantity, the value of $n$ which makes maximum the expression:

$$
\log \left|a_{n}\right|-\lambda_{n} \sigma
$$

will be one of those which satisfy (1.4.3). As a consequence of (1.4.2), we shall conclude that, if $\sigma$ is smaller than a negative quantity, in the circle (1.4.1) there will exist a point $s^{\prime}$ at which the following inequality will hold:

$$
\log \left|F\left(s^{\prime}\right)\right|>\log \mu\left(\sigma_{0}+d\right),
$$

where $d=D^{*}\left(7-3 \log \left(h D^{*}\right)\right)+\epsilon$. Moreover, according to Sugimura [8, Theorem 5], as $D^{*}<e \bar{D}^{*}$ is finite,

$$
\log \mu(\sigma)=(1-\epsilon(\sigma)) \log M(\sigma, F)
$$

and consequently we shall have

$$
\log \left|F\left(s^{\prime}\right)\right|>\left(1-\epsilon\left(\sigma_{0}\right)\right) \log M\left(\sigma_{0}+d, F\right)
$$

and for any finite value of $a$

$$
\log \left|F\left(s^{\prime}\right)-a\right|>\left(1-\epsilon\left(\sigma_{0}\right)\right) \log M\left(\sigma_{0}+d, F\right)
$$

because according to a result due to Doetsch [1], $M(\sigma, F)$ indefinitely increases as $\sigma$ tends to $-\infty$.

If $\bar{D}^{*}<1 / 2$ and if

$$
\limsup _{\sigma=-\infty} \frac{n(\sigma, Y, F-a)}{V(\sigma)}=B<B_{0}
$$

in any strip $Y^{\prime}$ defined by

$$
|t| \leqq \beta>\pi \max \left[\bar{D}^{*}, \frac{1}{2 \rho}\right],
$$

then, according to Lemma 1 , there exist a sequence of points 


$$
\left\{s_{n}=\sigma_{n}+i t_{n}\right\} \quad\left(\lim _{n=\infty} \sigma_{n}=-\infty,\left|t_{n}\right| \leqq \beta\right)
$$

and a quantity $r$ which depends only on $h, \rho$, and $Y^{\prime}$, that is to say, on $h, \rho$, and $\bar{D}^{*}$, such that in the circles:

$$
\left|s-s_{n}\right| \leqq r
$$

the following inequality holds:

$$
\log |F(s)-a|<0 .
$$

Evidently, if $\bar{D}^{*}<1 /(2 \rho)$, we shall be able to choose $Y^{\prime}$ fixed and independent of the value of $\bar{D}^{*}$; consequently, the quantities $B_{0}$ and $r$ will depend only on $h$ and $\rho$; that proves that, if

$$
\bar{D}^{*}<\min \left(\frac{1}{2 \rho}, \frac{r}{\pi}\right), \quad B<B_{0},
$$

inequality (1.4.4) in which we shall put $s_{0}=s_{n}$ and inequality (1.4.6) will be incompatible, because for a value of $\epsilon$ sufficiently small the circle (1.4.1) will lie entirely inside of (1.4.5); therefore, if

$$
\bar{D}^{*}<\Delta=\min \left(\frac{1}{2 \rho}, \frac{r}{\pi}\right)
$$

it will follow that $B \geqq B_{0}$, which is the statement of the theorem.

1.5. REMARKS. If

$$
\liminf _{\sigma=-\infty} \frac{\log _{2} M(\sigma, F)}{-\sigma}=\rho_{1}>0
$$

and if $\rho_{1}(\sigma)$ is a proximate order such that

$$
\lim _{\sigma=-\infty} \frac{V_{1}(\sigma)}{\log M(\sigma, F)}=0 \quad\left(V_{1}(\sigma)=e^{-\rho_{1}(\sigma) \sigma}\right),
$$

for any $f_{0}(s) \not \equiv 0$ and $f_{1}(s)$, entire and of proximate order $\leqq \rho_{1}(\sigma)$, then, with the hypothesis of Theorem I, the following relationship will hold:

$$
\limsup _{\sigma=-\infty} \frac{n\left(\sigma, Y, f_{0} F-f_{1}\right)}{V(\sigma)} \geqq B_{0} .
$$

Moreover, if the function $F(s)$ is of a very regular growth with respect to the proximate order $\rho(\sigma)$, that is to say, when the following relationship holds: 


$$
\liminf _{\sigma=-\infty} \frac{\log M(\sigma, F)}{V(\sigma)}=A_{1}>0,
$$

not only will the limit superior of the expression $n\left(\sigma, Y, f_{0} F\right.$ $\left.-f_{1}\right) / V(\sigma)$ have lower positive bound, but the same will happen with the limit inferior of this expression; that is to say, with the hypothesis of Theorem I, and on supposing (1.5.1) true, the relationship

$$
\liminf _{\sigma=-\infty} \frac{n\left(\sigma, Y, f_{0} F-f_{1}\right)}{V(\sigma)} \geqq B_{0}^{\prime}>0
$$

will hold, in which $B_{0}^{\prime}$ depends only on $Y, \rho, h$, and $A_{1}$.

Finally, on representing by $D$ the maximum density of $\left\{\lambda_{n}\right\}$, the inequalities $\bar{D}^{*} \leqq D^{*} \leqq D$ allow us to express the gap hypothesis in Theorem I and in the two precisions we have just indicated, by substituting $\bar{D}^{*}$ by $D^{*}$, or else by $D$. Moreover, if we express the gap condition by means of $D$, it is possible, by using a generalization of a result due to Pólya [7, chap. III, Theorem IV] instead of Mandelbrojt's inequality, to prove that in this case the quantities $\Delta$ and $B_{0}$ are independent of $h$, if $h>0$.

\section{2}

2.1. In a way similar to that of $\$ 1.1$, we shall consider an entire function $f(s)$ which satisfies the following condition:

$$
\underset{-\infty<i<+\infty}{\text { l.u.b. }}|f(\sigma+i t)|=M(\sigma, f), \quad \underset{\sigma 1 \leqq \sigma \leqq \sigma_{2}}{\text { l.u.b. }} M(\sigma, f)<\infty
$$

for any $\sigma_{1}$ and $\sigma_{2}$. But contrary to the hypothesis of 1.1 , we shall suppose that

$$
\limsup _{\sigma=-\infty} \frac{\log _{2} M(\sigma, f)}{-\sigma}=\infty,
$$

that is to say, we shall suppose that $f(s)$ is of infinite order $(R)$.

Following a procedure similar to that of K. L. Hiong [2], it may be proved that in this case there always exist functions $W(\sigma)$ with the following properties:

$$
\begin{gathered}
W\left(\sigma-\frac{1}{\log W(\sigma)}\right)<[W(\sigma)]^{1+\epsilon(\sigma)}, \\
\limsup _{\sigma=-\infty} \frac{\log _{2} M(\sigma, f)}{\log W(\sigma)}=1
\end{gathered}
$$


and finally $\log W(\sigma)$ is a convex function of $\sigma$. Any function with the above properties shall be called an order of $f(s)$. (Generally it is the expression $(\log W(\sigma)) /-\sigma$ which receives that name, but owing to various reasons we shall not follow the custom.)

Likewise, if $f(s)$ is of infinite order in the strip $Y$, there will exist functions $W(\sigma)$ with the same properties as before, but, instead of satisfying (2.1.1) they will satisfy the relationship:

$$
\limsup _{\sigma=-\infty} \frac{\log \log ^{+} M(\sigma, Y, f)}{\log W(\sigma)}=1,
$$

and any function of this class shall be called an order of $f(s)$ in $Y$.

\subsection{ThEOREM II. Let}

$$
F(s)=\sum_{n=0}^{\infty} a_{n} e^{-\lambda_{n}}
$$

be a Dirichlet series convergent throughout the plane and which represents a function of order $W(\sigma)$.

If the sequence $\left\{\lambda_{n}\right\}$ has the properties:

$$
\underset{\lambda=\infty}{\limsup } D(\lambda) \lambda^{\beta}<\infty \quad(0<\beta<1), \quad \lim _{n=\infty} \inf \left(\lambda_{n+1}-\lambda_{n}\right)=h>0,
$$

whatever the strip $Y$ and the finite value $a$ (without exception) may be, it follows that

$$
\limsup _{\sigma=-\infty} \frac{\log n(\sigma, Y, F-a)}{\log W(\sigma)}=1 .
$$

2.3. Similar to the reasoning in the case of finite order, the proof of the preceding theorem may be based on the following lemma.

LEMMA 2. Let $Y$ be any strip and $Y^{\prime}$ another strip contained in $Y$. If $W(\sigma)$ is an order of $f(s)$ both in $Y$ and in $Y^{\prime}$, and if

$$
\limsup _{\sigma=-\infty} \frac{\log n(\sigma, Y, f)}{\log W(\sigma)}<1,
$$

there will exist a sequence $\left\{s_{n}=\sigma_{n}+i t_{n}\right\}\left(\lim \sigma_{n}=-\infty\right)$ of points inside $Y$ such that in the circles

$$
\left|s-s_{n}\right| \leqq\left[W\left(\sigma_{n}\right)\right]^{-\gamma},
$$

where $\gamma>0$ is arbitrary, the relationship

$$
\log |f(s)|<-\left[W\left(\sigma_{n}\right)\right]^{1-\epsilon\left(\sigma_{n}\right)}
$$

holds. 
The proof of this lemma is almost the same as that of Lemma 1.

2.4. According to a result of Mandelbrojt which we have made use of, in the circle

$$
\left|s-s_{0}\right| \leqq r \quad\left(s_{0}=\sigma_{0}+i t_{0}\right)
$$

there exists a point $s^{\prime}$ at which ${ }^{6}$

$$
\log \left|F\left(s^{\prime}\right)\right|>\log \left|a_{n}\right|-\lambda_{n} \sigma_{0}-\log \Lambda_{n}^{*}-\log (r L(r))
$$

for any value of $n$. Taking the value of $n$ which gives the maximum value to the expression

$$
\log \left|a_{n}\right|-\lambda_{n} \sigma_{0}
$$

the above inequality will take the form:

$$
\log \left|F\left(s^{\prime}\right)\right|>\log \mu\left(\sigma_{0}\right)-\log \Lambda_{n}^{*}-\log (r L(r)) .
$$

On the other hand, it is easy to prove that, in the Dirichlet series having the properties we are now taking into account, the relationship

$$
M(\sigma, F)<\mu(\sigma)[W(\sigma)]^{1+\epsilon(\sigma)}
$$

holds, and that from this inequality it follows that if $\left\{\sigma_{k}\right\}$ possesses the properties:

$$
\lim _{k=\infty} \sigma_{k}=-\infty, \quad \log M\left(\sigma_{k}, F\right)>\left[W\left(\sigma_{k}\right)\right]^{1-\epsilon\left(\sigma_{k}\right)},
$$

then it will possess the property:

$$
\log \mu\left(\sigma_{k}\right)>\left[W\left(\sigma_{k}\right)\right]^{1-\epsilon\left(\sigma_{k}\right)} .
$$

Moreover, if we represent by $\lambda(\sigma)$ the value of $\lambda_{n}$ corresponding to the value of $n$ which gives the maximum value to the expression $\log \left|a_{n}\right|-\lambda_{n} \sigma$, then it is possible to prove that

$$
\lambda(\sigma)<[W(\sigma)]^{1+\epsilon(\sigma)}
$$

and, therefore, according to Mandelbrojt $[4$, p. 355] we shall have in (2.4.1)

$$
\log \Lambda_{n}^{*}<\left[W\left(\sigma_{0}\right)\right]^{(1-\beta)\left(1+\epsilon\left(\sigma_{0}\right)\right)} .
$$

Likewise, according to Mandelbrojt [5, Lemma VI], from the condition

$$
\limsup _{\lambda=\infty} D(\lambda) \lambda^{\beta}<\infty
$$

- Since $L_{n}(r)<L(r)=\int_{0}^{\infty} \Lambda(t) e^{-t r} d t$, the $L_{n}(r)$ can be replaced by $L(r)$. 
it follows that

$$
\log (r L(r)) \leqq C r^{(\beta-1) / \beta}
$$$$
(C=\text { constant }<\infty) \text {. }
$$

As a consequence, for any $t_{0}$, if we put

$$
\sigma_{0}=\sigma_{k}, \quad r=\left[W\left(\sigma_{k}\right)\right]^{-\theta} \quad(\theta<\beta /(1-\beta)),
$$

it follows from (2.4.1), (2.4.2), (2.4.3), and (2.4.4) that the function $F(s)$ is of order $W(\sigma)$ in any strip, and evidently, the same happens to the function $F(s)-a$.

If, contrary to the statement of the theorem, there were a finite value $a$ and a strip $Y$ for which

$$
\limsup _{\sigma=-\infty} \frac{\log n(\sigma, Y, F-a)}{\log W(\sigma)}<1,
$$

then, from Lemma 2, there would exist a sequence of points $\left\{s_{k}^{\prime}=\sigma_{k}^{\prime}+i t_{k}^{\prime}\right\}$ inside $Y$, such that in the circles

$$
\left|s-s_{k}^{\prime}\right| \leqq\left[W\left(\sigma_{k}^{\prime}\right)\right]^{-\gamma} \text {, }
$$

the relationship

$$
\log |F(s)-a|<-\left[W\left(\sigma_{k}^{\prime}\right)\right]^{1-\epsilon\left(\sigma_{k}^{\prime}\right)}
$$

will hold.

On the other hand, applying to $F(s)-a$ Mandelbrojt's result which we have made use of several times, but keeping constant the value of $n=n_{0}$ in such a way that $a_{n_{0}} \neq 0$, it will follow that, in the circles

$$
\left|s-s_{k}^{\prime}\right| \leqq r=\left[W\left(\sigma_{k}^{\prime}\right)\right]^{-\theta} \quad(\theta<\beta /(1-\beta)),
$$

there will exist a point $s^{\prime \prime}$ at which

$$
\begin{aligned}
\log \left|F\left(s^{\prime \prime}\right)-a\right| & >\log \left|a_{n_{0}}\right|-\lambda_{n_{0}} \sigma_{k}^{\prime}-\log \Lambda_{n_{0}}^{*} \\
& -\log (r L(r))>-C\left[W\left(\sigma_{k}^{\prime}\right)\right]^{\theta(1-\beta) / \beta}
\end{aligned}
$$

and since the value of $\gamma$ in Lemma 2 is arbitrary, we may suppose that $\gamma<\theta$ and, as a consequence the inequalities (2.4.5) and (2.4.6) are incompatible, which proves the theorem.

2.5. As in the case of finite order, when the function $F(s)$ is of a regular growth with respect to $W(\sigma)$, that is to say, when

$$
\lim _{\sigma=-\infty} \frac{\log _{2} M(\sigma, F)}{\log W(\sigma)}=1,
$$

Theorem II may be stated in the following way:

If the hypotheses of Theorem II are fulfilled and $F(s)$ is of a regular 
growth with respect to $W(\sigma)$, for any $f_{0}(s) \not \equiv 0$ and $f_{1}(s)$, entire and of respective orders $W_{0}(\sigma)$ and $W_{1}(\sigma)$ such that

$$
\begin{array}{lr}
W_{0}(\sigma)<[W(\sigma)]^{b} & (b<\min (1, \beta /(1-\beta))), \\
W_{1}(\sigma)<[W(\sigma)]^{b_{1}} & \left(b_{1}<1\right),
\end{array}
$$

we shall have

$$
\lim _{\sigma=-\infty} \frac{\log n\left(\sigma, Y, f_{0} F-f_{1}\right)}{\log W(\sigma)}=1
$$

whatever the strip $Y$ may be.

\section{BIBLIOGRAPHY}

1. G. Doetsch, Über die obere Grenze des absoluten Beitrages einer analytischen Funktion auf Geraden, Math. Zeit. vol. 8 (1920) p. 237.

2. K. L. Hiong, Sur les fonctions entieres et les fonctions meromorphes d'ordre infini, Journal de Mathematiques (9) vol. 14 (1935) p. 233.

3. S. Mandelbrojt, Dirichlet series, The Rice Institute Pamphlet, vol. 31, No. 4, 1944.

4. - Sur une inegalite fondamentale, Ann. Ecole Norm. (3) vol. 63 (1947) p. 351.

5. - Quasi-analyticity and analytic continuation-a general principle, Trans. Amer. Math. Soc. vol. 55 (1944) pp. 96-131.

6. R. Nevanlinna, Eindeutige analytische Funktionen, Berlin, 1936.

7. G. P6lya, Untersuchungen ubber Lucken und Singularitäte von Potensreihen, Math. Zeit. vol. 29 (1929) p. 549.

8. K. Sugimura, Ubbertragung einiger Satze ans der Theorie der ganzen Funktionen auf Dirichletschen Reihen, Math. Zeit. vol. 29 (1929) p. 264.

9. F. Sunyer i Balaguer, Sur la substitution d'une valeur exceptionnelle par une propriete lacunaire, C. R. Acad. Sci. Paris vol. 224 (1947) pp. 1609-1611 and vol. 225 (1947) pp. 21-23; for the proofs see Acta Math. vol. 87 (1952) pp. 17-31.

10. - Propiedades de las funciones enteras representadas por series de Taylor lagunares (orden finito), Collectanea Mathematica vol. 2 (1949) p. 129.

11. V. Bernstein, Sulle direcioni di Borel di funsioni olomorfe, Annali di Mat. (4) vol. 12 (1934).

UNIVERSITY ÓF BARCELONA 